\title{
2 Patienteninformation
}

Julia Bellabarba

„Es gibt keine Wahrheit. Nur noch Verantwortung“, hat Heinz von Foerster gesagt. Was hat das mit unserem Thema zu tun? Patienten in Deutschland sind, im internationalen Vergleich, unzufrieden mit der Kommunikationsfähigkeit der Ärzte. Der Commonwealth Fund führte 2007 eine Befragung zur Zufriedenheit mit dem Gesundheitswesen in sieben Ländern durch (Australien, Kanada, Deutschland, Niederlande, Neu Seeland, Großbritannien, USA). Deutsche Patienten beklagten Mängel besonders bei der Koordinierung der Schnittstellen, bei der Information, Kommunikation und bei der Aufklärung durch die Ärzte. Die Verantwortung, dies zu ändern, liegt bei Ihnen.

„Der Arzt hat den Patienten rechtzeitig vor der Behandlung und grundsätzlich in einem persönlichen Gespräch über Art und Umfang der Maßnahmen und der damit verbundenen gesundheitlichen Risiken aufzuklären und die Einwilligung des Patienten dazu einzuholen. Formulare und Aufklärungsbögen ersetzen das Gespräch nicht. (...) Auf Fragen des Patienten hat der Arzt wahrheitsgemäß, vollständig und verständlich zu antworten. Aufklärung und Beratung müssen auch für Patienten, die sich mit dem Arzt sprachlich nicht verständigen können, verstehbar sein." (Patientenrechte in Deutschland, S. 10)

Für die Patienten in Deutschland ist der Arzt die wichtigste Informationsquelle zum Thema Gesundheit. Zu den Themen Medikation und Prävention ist der Arzt für viele Patienten sogar die einzige Informationsquelle (Makoul et al. 1995). Diercks et al. (2000) erhoben die Patientenwünsche in einer Befragung von knapp 1000 Patienten zwischen 18 und 80 Jahren in Praxen niedergelassener Haus- und Fachärzte. Obwohl über zwei Drittel der Befragten, das Bedürfnis nach Informationen als „sehr wichtig“ einstuften, waren nur $50 \%$ dieser 
Patienten „sehr zufrieden“ mit den erhaltenen Informationen. Bei den Ärzten nimmt die Aufgabe, Patienten zu informieren, einen geringeren Stellenwert ein, als von den Patienten gewünscht wird (Laine et al. 1996, Makoul et al. 1995, Baum et al. 1996). Dabei zeigt eine Übersichtsarbeit schon vor mehr als 10 Jahren (Segal 1998), dass der Faktor „Information“ nicht nur wesentlich zur Patientenzufriedenheit beiträgt, sondern positive Effekte auch auf den Gesundheitszustand zeigt: Bei chronischen Schmerzpatienten, bei Diabetikern, Rheumatikern und Patienten mit chronischen psychischen Problemen sowie bei Patienten mit schweren Kopfverletzungen konnte durch eine verbesserte Informations- und Entscheidungsbeteiligung auch eine Verbesserung des Gesundheitszustand erreicht werden.

Im $₫ 3$ der QM-Richtlinie „Grundelemente eines einrichtungsinternen Qualitätsmanagements“ steht Patienteninformation (neben Patientenberatung, -orientierung, -sicherheit und -mitwirkung) an zentraler Stelle. Eine umfassende Information des Patienten zu diagnostischen Maßnahmen und zum Behandlungsverlauf wird also vom Arzt sowie vom Psychotherapeuten gefordert. Der Zielsetzung dieses Buches entsprechend, möchte ich das Thema Patienteninformation möglicht praxisnah anhand der folgenden drei Fragestellungen diskutieren.

\subsection{Patientenaufklärung}

Fragen an die Leser: Zu welchen Informationen sind Sie gesetzlich verpflichtet? Welche Patienteninformationen halten Sie darüber hinaus für unabdingbar?

- zur Erkrankung

- zur Medikation

- zu Behandlungsalternativen

- zu Eingriffen

- zu Prävention

- zur Praxisorganisation

Den behandelnden Arzt oder Psychologen trifft im Zusammenhang mit der Patientenaufklärung die Beweispflicht, d.h. er muss nachweisen, ausreichend aufgeklärt zu haben (oder dies veranlasst zu haben).

„Die höchstrichterliche Rechtssprechung zur Patientenaufklärung führte zu einer enormen Haftungsausweitung, weshalb jede Ärztin und jeder Arzt angesichts des daraus resultieren forensischen Risikos im Zusammenhang mit Aufklärungsmaßnahmen ... zu besonderer Sorgfalt verpflichtet ist.“ (Bock 2009, S. 17)

Die Pflicht zur adäquaten Patienteninformation sollte jedoch nicht in erster Linie unter formalrechtlichen Aspekten betrachtet werden, oder als lästige Formalität delegiert werden, denn diese Informationsgespräche enthalten immer auch ein Beziehungsangebot auf dessen Grundlage Akzeptanz und so- 
mit Compliance beruht. Im Zusammenhang mit der Aufklärungspflicht des Arztes muss auch das Selbstbestimmungsrecht des Patienten bedacht und organisatorisch umgesetzt werden. Es ist also sinnvoll, im Praxisteam und auch als allein praktizierender Psychotherapeut zu überlegen, durch welche organisatorischen Maßnahmen der Patient in seinem Entscheidungsprozess unterstützt wird, und wie dies dokumentiert wird (vgl. auch Kap. V.3).

\subsection{Empfehlungen}

Frage an die Leser: Welche Informationsquellen (Internet, Broschüren, Bücher, Selbsthilfegruppen) möchten Sie Ihren Patienten empfehlen oder mitgeben?

Zu den häufigsten oder schwerwiegenden Erkrankungen sollte die Praxis selbst Informationen geben und gezielt auf Informationsquellen hinweisen. Es wird zunehmend wichtiger, den Markt der Patienteninformationen im Internet zu sichten und Patienten auf vertrauenswürdige, gesicherte Links hinzuweisen. Die Aufklärung bei Patienten ohne deutsche Sprachkompetenz kann wirksam durch muttersprachliches Material unterstützt werden. Unter den unten aufgeführten Links können sich Ärzte informieren, welche (qualitätsgeprüften, unabhängigen) Informationen aktuell für Patienten zur Verfügung stehen. Die unkritische Weitergabe von Broschüren, die von PharmaUnternehmen erstellt werden, ist als problematisch zu betrachten.

„Medikamentenwerbung an Patienten und Bürger für rezeptpflichtige Medikamente durch die Industrie unter dem Deckmantel der Information hat erhebliche Nebenwirkungen jedoch keinen nachweisbaren Nutzen“ (Mühlhauser und Lenz 2008, S. 223).

\subsection{Eigene Information}

Frage an die Leser: Welche Informationen möchten Sie selber erstellen, um sich den Arbeitsablauf zu erleichtern?

Die meisten Ärzte und medizinischen Fachangestellten führen jeden Tag zeitraubende Gespräche, in denen unzählige Male dasselbe erläutet werden muss. Ausgesprochen hilfreich ist es, wenn Praxen selbst erstelltes Informationsmaterial für Patienten haben. Fragen Sie sich: Welche Informationen wiederholen wir im Laufe des Tages immer wieder? Wo entstehen Missverständnisse mit den Patienten? Wo möchten wir eine bessere Compliance erreichen? Überlegen Sie im Team, welche Informationen Sie zusammenfassen möchten, um sie Patienten mitgeben zu können. Erstellen Sie kurze Informationsblätter zu den Themen, achten Sie auf Verständlichkeit. Diese Zettel können ganz einfache, praktische Tipps enthalten, die der Patient immer wieder durchlesen kann, und für Sie einen erheblichen Zeitgewinn und eine größere Sicherheit in Hinblick auf die Umsetzung der ärztlichen Empfehlungen darstellen. 


\section{Praxisbeispiel}

Eine HNO-Praxis gibt ihren Patienten Zettel mit Informationen zu folgenden Fragen mit:

- Bei Halsschmerzen: Wie mache ich einen Halswickel richtig?

- Bei Husten und Heiserkeit: Wie inhaliere ich richtig?

- Bei gereiztem oder juckendem Gehörgang: Pflegende Behandlung des Gehörganges

- Bei leichten Ohrenschmerzen: Zwiebelpackungen zur Wärmebehandlung des Ohres bei Reizungen.

- Zur Nasenspülung: Wie stelle ich eine Salzlösung richtig her?

- Bei verstopfter Nase: Wie führe ich eine Nasenspülung richtig durch?

- Bei Reizhusten: Wie wende ich einen Brustwickel richtig an?

- Bei Fieber: Wie mache ich einen Wadenwickel zur Fiebersenkung richtig?

\section{Literatur}

Baum E, Donner-Banzhoff N, Spangenberg E, Platt B: Erwartungen der Patienten und ärztliches Handeln in Allgemeinarztpraxen. In: Lang E, Arnold K (Hrsg.): Die Arzt-Patient-Beziehung im Wandel. Schriftenreihe der Hamburg-Mannheimer-Stiftung für Informationsmedizin. Stuttgart: Enke Verlag 1996; 137-150

Bock R-W: Recht für Krankenhaus und Arztpraxis. Berlin: Medizinisch Wissenschaftliche Verlagsgesellschaft 2009

Dierks ML, Schwartz FW, Walter U: Patienten als Kunden. Informationsbedarf und Qualität von Patienteninformationen aus Sicht der Public Health-Forschung. In: Jazbinsek D (Hrsg.): Gesundheitskommunikation. Wiesbaden: Westdeutscher Verlag 2000: 150-163

Makoul G, Arntson P, Schofield Th: Health Promotion in Primary Care: Physician-Patient Communication and Decision Making about Prescription Medications. Social Science and Medicine 1995; 41 (9): 1241-1254

Laine $C$ et al.: Important Elements of Outpatient Care: A Comparison of Patients' and Physicians' Opinions. Annals of Internal Medicine 1996; 125 (8): 640-645

Mühlhauser I, Lenz M: Verbesserung der Therapieergebnisse durch Patientenwissen? Z Evid Fortbild Qual Gesundh.wesen 2008; 102: 223-230

Sänger S, Lang B, Klemperer D, Thomeczek C, Dierks ML: Manual Patienteninformation - Empfehlungen zur Erstellung evidenzbasierter Patienteninformationen. Berlin: Ärztliches Zentrum für Qualität in der Medizin (ÄZQ) 2006

Segal L: The Importance of Patient Empowerment in Health System Reform. Health Policy 1998; 44: 31-44 Steckelberg A, Berger B, Kopke S, Heesen C, Muhlhauser I: Kriterien für evidenzbasierte Patienteninformation. Z Ärztl Fortbild Qualitätssich 2005; 99(6): 343-51

\section{links}

www.gesundheitsinformation.de/index.de.html

www.patientenleitlinien.de

www.akdae.de/45/index.html

www.patienten-information.de 
Die Autorin hat Patienteninformationen für Kinder und Jugendliche zu folgenden Themen erstellt:

- ADHS: Meine Konzentration und ich.

- Enuresis (dtsch./türk.): Mein trockenes Bett und ich.

- Kinder-und Jugendpsychiatrische Behandlung allgemein: Mein Kinder- und Jugendpsychiater und ich.

Interessenten können sich unter info@quipps.de melden. 